УДК 518.6, 536.6

\title{
The Calculation of the Spatial Distribution of Temperature Fields for Remote Monitoring of the Surface From an Unmanned Aerial Vehicle
}

\author{
Igor N. Ischuk ${ }^{b}$, Alexey A. Dolgov', \\ Andrew A. Bebenin a and Sergey A. Panova \\ ${ }^{a}$ Military Education and Research Centre of Military-Air Forces \\ «Military-Air Academy \\ Named After Professor N.E. Zhukovsky and Yu.A. Gagarin» \\ 54a Starykh Bol'shevikov Str., Voronezh, 394064, Russia \\ ${ }^{b}$ Siberian Federal University \\ 79 Svobodny, Krasnoyarsk, 660041, Russia
}

The article deals with the calculation of the spatial distribution of temperature fields on the surface and deep into the soil. Presents a general formulation of the problem of calculating the spatial distribution of temperature fields in variably-saturated porous media. The results of the proposed method of calculation in the field experiment.

Keywords: orthotropic medium, variably-saturated porous medium, temperature field, temperature distribution, heat flux, mathematical and software simulation.

Citation: Ischuk I.N., Dolgov A.A., Bebenin A.A., Panov S.A. The calculation of the spatial distribution of temperature fields for remote monitoring of the surface from an unmanned aerial vehicle, J. Sib. Fed. Univ. Eng. technol., 2018, 11(3), 273-279. DOI: 10.17516/1999-494X-0039.

(c) Siberian Federal University. All rights reserved

* Corresponding author E-mail address: boerby@rambler.ru; alexdolgov88-08@rambler.ru 


\title{
Расчет пространственного распределения \\ температурных полей \\ при дистанционном мониторинге поверхности территорий \\ с беспилотного летательного аппарата
}

\author{
И.Н. Ищук \\ А.А. Бебенин ${ }^{\text {a }}$ С.А. Панов ${ }^{\text {a }}$ \\ ${ }^{a}$ Военный учебно-научный иеентр Военно-воздушных сил \\ «Военно-воздушная академия \\ имени профессора Н.Е. Жуковского и Ю.А. Гагарина» \\ Россия, 394064, Воронеж, ул. Старых Большевиков, 54 а \\ ${ }^{\sigma}$ Сибирский федеральный университет \\ Россия, 660041, Красноярск, пр. Свободньй, 79
}

\begin{abstract}
В статье рассматривается расчет пространственного распределения температурньх полей на поверхности и вглубь грунта. Представлена общая постановка задачи расчета пространственного распределения температурных полей в переменно-насыщенных пористых средах. Приведены результаты реализации предложенного способа расчета в ходе натурного эксперимента.
\end{abstract}

Ключевые слова: ортотропная среда, переменно-насыщенная пористая среда, температурное поле, распределение температур, поток тепла, математическое и программное моделирование.

\section{Введение}

Одной из перспективных технологий мониторинга и анализа техногенных территорий с применением беспилотных летательных аппаратов (БПЛА) большой длительности полета является усвоение экспериментальных многоспектральных динамических данных наблюдения. Весьма важную роль играет практическое использование получаемых оценок двумерного пространственного распределения теплофизических параметров поверхностного и глубинного слоев территории мониторинга, а также получаемых тепловых томограмм этих слоев. Рассмотрим постановку задачи и расчет пространственного распределения температурных полей в негомогенной ортотропной среде (почве) с применением программного пакета математического моделирования одномерного движения воды, тепла и множества растворов в переменнонасыщенных средах HYDRUS1D.

\section{1. Постановка задачи расчета пространственного распределения температурных полей в переменно-насыщенных пористых средах}

Распределение температур в анизотропной переменно-насыщенной пористой среде с разрывными коэффициентами в условиях теплообмена на границе с окружающей средой исследуется на основе решения краевой задачи для конвекционно-диффузионного уравнения [1]: 


$$
\begin{aligned}
& C_{\rho j}(\theta) \frac{\partial T_{j}}{\partial \tau}=\frac{\partial}{\partial x}\left(\left[\lambda_{j}(\theta)\right]_{11} \frac{\partial T_{j}}{\partial x}\right)+2 \frac{\partial}{\partial x}\left(\left[\lambda_{j}(\theta)\right]_{12} \frac{\partial T_{j}}{\partial y}\right)+2 \frac{\partial}{\partial x}\left(\left[\lambda_{j}(\theta)\right]_{13} \frac{\partial T_{j}}{\partial z}\right)+ \\
& +\frac{\partial}{\partial y}\left(\left[\lambda_{j}(\theta)\right]_{22} \frac{\partial T_{j}}{\partial y}\right)+2 \frac{\partial}{\partial y}\left(\left[\lambda_{j}(\theta)\right]_{23} \frac{\partial T_{j}}{\partial z}\right)+\frac{\partial}{\partial z}\left(\left[\lambda_{j}(\theta)\right]_{33} \frac{\partial T_{j}}{\partial z}\right)-C_{w j} \frac{\partial T_{j}}{\partial y}
\end{aligned}
$$

где $T=T(x, y, z, \tau)$ - неизвестная функция температуры, $\tau \in\left[\tau_{0}, \tau_{1}\right],(x, y, z) \in D=\Omega \times[0, H]$, $\Omega \subset R^{2}, \Omega$ - поверхность полуограниченной среды; $C_{r}, C_{w}-$ теплоемкости пористой среды и жидкости соответственно; $\left[\lambda_{j}(\theta)\right]_{11},\left[\lambda_{j}(\theta)\right]_{12},\left[\lambda_{j}(\theta)\right]_{13},\left[\lambda_{j}(\theta)\right]_{22},\left[\lambda_{j}(\theta)\right]_{13},\left[\lambda_{j}(\theta)\right]_{33}-$ компоненты тензора эффективной теплопроводности негомогенной пористой среды (почвы) с разрывными коэффициентами $(j \in[0, H])$ в зависимости от потенциальной температуры $\theta$. Она может быть описана экспериментальной зависимостью [2]: $\lambda(\theta)=b_{1}+b_{2} \theta+b_{3} \theta^{\frac{1}{2}}$, где $b_{1}, b_{2}, b_{3}$ - эмпирические параметры среды (почвы). Также в альтернативе при описании эффективной теплопроводности можно использовать зависимость $\lambda(\theta)=A+B \theta-(A-D) \exp \left[-(C \theta)^{E}\right]$, где $A=\frac{0.57+1.73 \theta_{q}+0.93 \theta_{m}}{1-0.74 \theta_{q}-0.49 \theta_{m}}-2.8 \theta_{n}\left(1-\theta_{n}\right), B=2.8 \theta_{n}, C=1+2.6 \theta_{n}^{-\frac{1}{2}}, D=0.03+0.7 \theta_{n}^{2}, E=4$, где индексы $n, q, c$ и $m$ обозначают почву, кварц, глину и другие минералы.

Краевые условия, учитывающие уравнение теплового баланса и сопряжения слоистых сред, будут выглядеть следующим образом:

$$
\begin{aligned}
& -\left.C^{*} \rho k \frac{\partial T_{0}}{\partial z}\right|_{z=0}-\left.L_{\rho} k \frac{\partial s}{\partial z}\right|_{z=0}-\left.\lambda_{1}(\theta) \frac{\partial T_{0}}{\partial z}\right|_{z=0}-B_{0}^{*}=q_{E}-\left.C_{v} \frac{\partial q_{v} T_{0}}{\partial z}\right|_{z=0}-\left.L_{\rho} \frac{\partial q_{v}}{\partial z}\right|_{z=0} ; \\
& \left.\lambda_{j}(\theta) \frac{\partial T_{j}}{\partial z}\right|_{z=-h_{j}}=\frac{1}{R_{T}}\left(\left.T_{j+1}\right|_{z=+h_{j}}-\left.T_{j}\right|_{z=-h_{j}}\right) ; \\
& \left.\lambda_{j}(\theta) \frac{\partial T_{j}}{\partial z}\right|_{z=-h_{j}}=\left.\lambda_{j+1}(\theta) \frac{\partial T_{j+1}}{\partial z}\right|_{z=+h_{j}} ; \\
& \left.T_{N}\right|_{z=H}=\varphi_{1}(x, y), \\
& \left.T_{0}\right|_{z=0}=\varphi_{0}(x, y),
\end{aligned}
$$

где $C^{*}$ - удельная изобарная теплоемкость воздуха; $\rho$ - плотность воздуха; $k$ - коэффициент турбулентности; $s$ - массовая доля водяного пара; $L$ - удельная теплота парообразования; $B_{0}^{*}=\delta\left(\sigma T_{0}^{4}-\delta_{a} \sigma T_{I}^{4}\right)$ - эффективное излучение верхнего слоя поверхности; $\delta$ - поглощательная способность верхнего слоя поверхности; $\sigma$ - постоянная Стефана - Больцмана; $q$ - плотность теплового потока; $C_{v}$ - удельная теплоемкость водяного пара; $R_{T}$ - коэффициент термического сопротивления; $H$ - координата нижней границы; $\varphi_{0}(x, y) ; \varphi_{1}(x, y)$ - известные функции начального распределения температур. В уравнении (2) суммарный приток тепла $Q=Q(x, y, z, \tau)$ определяется как сумма потока тепла излучением $q_{E}$ и конвекцией тепла водой, водой и паром, потоком пара $-q_{v}[1]: Q=q_{E}+q_{v}$.

Величина $q_{E}$ определяется в основном притоком солнечной радиации. Распределение солнечной радиации при отсутствии атмосферы (на верхней границе атмосферы) и изменение ее во времени зависят чисто от астрономических факторов: вращения Земли вокруг Солнца, наклона оси вращения Земли по отношению к плоскости эклиптики, суточного вращения Земли [3]. 
Количество солнечной радиации $q_{E}$, приходящейся на $1 \mathrm{~cm}^{2}$ горизонтальной поверхности в течение суток при условии отсутствия атмосферы, может быть найдено из уравнения

$$
q_{E}=F_{0} \int_{-t_{0}}^{t_{0}}\left(\sin \varphi \sin \xi+\cos \varphi \cos \xi \sin \frac{2 \pi}{P} t\right) \frac{d t}{R^{2}},
$$

где $F_{0}=1.88-1.98 \mathrm{Kaл}^{*} \mathrm{~cm}^{2} /$ мин - астрономическая солнечная постоянная; $\pm t_{0}-$ время восхода и захода Солнца, отсчитывая от полудня; $R=r / r_{0}-$ отношение расстояний произвольного и среднего расстояний от Земли до Солнца; $\varphi$ - широта места наблюдения; $\xi$ - угол склонения Солнца, зависящий от времени года $\left(-23^{0} 27^{\prime} \ldots+23^{0} 27^{\prime}\right), P$ - период вращения Земли; $\frac{2 \pi}{P} t$ часовой угол; $t$ - время, отсчитываемое от полудня [4].

Распределение солнечной радиации $q_{E}$ по часам в течение суток можно описать следующим образом:

$$
\Delta q_{j E}=\frac{q_{E}}{\sin \gamma}\left(\cos \left(\frac{2 \gamma \Delta \tau_{j}}{\tau}\right)-\cos \left(\frac{2 \gamma \Delta \tau_{j-1}}{\tau}\right)\right),
$$

где $\gamma$ - максимальный угол подъема Солнца над горизонтом в течение суток; $q_{E}-$ количество солнечной радиации за сутки; $\tau$ - продолжительность дня; $\left[\Delta \tau_{j-1} ; \Delta \tau_{j}\right]-$ временной интервал распределения солнечной радиации $\Delta q_{j} ; j \in[1, \tau]$ количество часов от восхода Солнца.

Плотность потока тепла жидкости и пара $q_{v}$ может быть описана выражением [1]

$$
q_{v}=\left(K+K_{v h}\right)\left(\frac{\partial p}{\partial z}+\cos \alpha\right)+\left(K_{L T}+K_{v T}\right) \frac{\partial T}{\partial z}
$$

где $K$ - изотермическая влагопроводность жидкости; $K_{v h}-$ изотермическая паровлагопроводность; $p$ - интенсивность потока, $\alpha$ - параметр в функции удержания грунтовых вод, $K_{L T}-$ тепловлагопроводность жидкости.

Границы области $\Gamma \equiv \partial D$ представляются как объединение непересекающихся частей $\Gamma_{0}=\Omega$ поверхности среды (почвы), $\Gamma_{H}$ - ограниченной глубины почвы, при этом $h_{0}(x, y), h_{H}(x, y)$ - функции профиля среды на соответствующей глубине z.

\section{2. Математическое моделирование распределения температурных полей поверхности при дистанционном мониторинге с БПЛА}

Решение рассматриваемой задачи возможно с помощью различных сред математического моделирования и программирования, таких как SolidWorks, TAITherm, Comsol, Flow3D, HYDRUSX1D.

Для решения данной задачи наиболее целесообразным является использование пакета математического моделирования одноразмерного движения воды, тепла и множества растворов в переменно-насыщенных средах HYDRUS1D, так как он позволяет рассчитывать распределения температурных полей вглубь поверхности почвы; учитывать состояние почвы (влажность), а также метеорологические условия проведения эксперимента. Совместное использование данного пакета с дополнительными модулями математической обработки данных среды MATLAB

$$
-276-
$$


позволяет получать распределения температурных полей по всей поверхности почвы в течение заданного временного интервала.

Для апробации расчета распределения температурных полей с помощью вышеописанного программного обеспечения был проведен натурный эксперимент, заключавшийся в измерении температуры поверхностного слоя известного типа грунта (глина) в течение суток с периодичностью 1 ч. Также с данной периодичностью фиксировались метеорологические условия: температура воздуха, измеренная на высоте 2 м от земной поверхности, скорость ветра, облачность и влажность [4]. В результате эксперимента всего было получено по 25 значений каждого из вышеуказанных параметров.

Приток солнечной энергии и его распределение в течение суток были рассчитаны аналитически с помощью уравнений (3) и (4) (рис. 1).

В результате моделирования был произведен расчет распределения температурного поля грунта на его поверхности и в глубину до 1 м. Результаты расчета приведены на рис. 2 и 3.

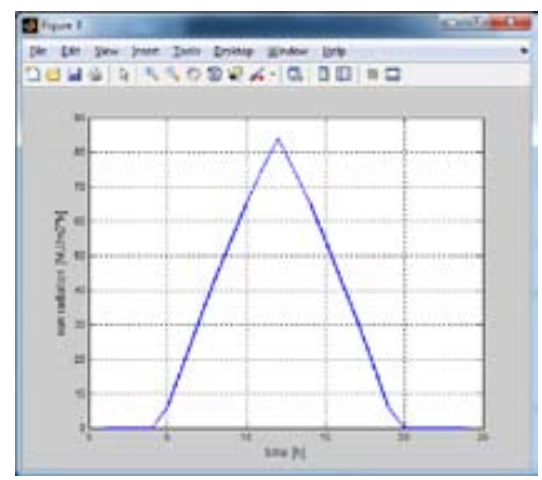

Рис. 1. Суточный ход солнечной радиации

Fig. 1. The daily course of solar radiation

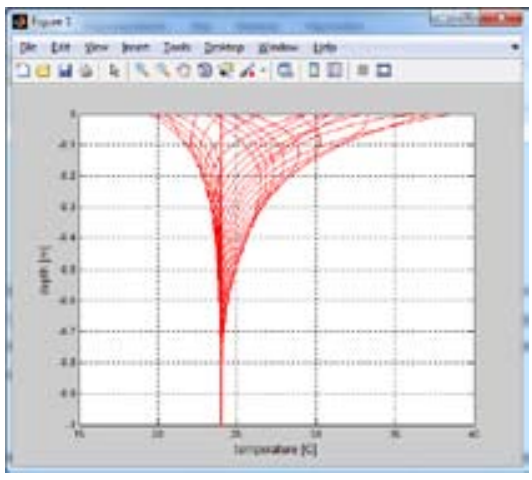

Рис. 2. Распределение температурного поля в глубину профиля грунта

Fig. 2. The distribution of temperature field in the depth of the soil profile

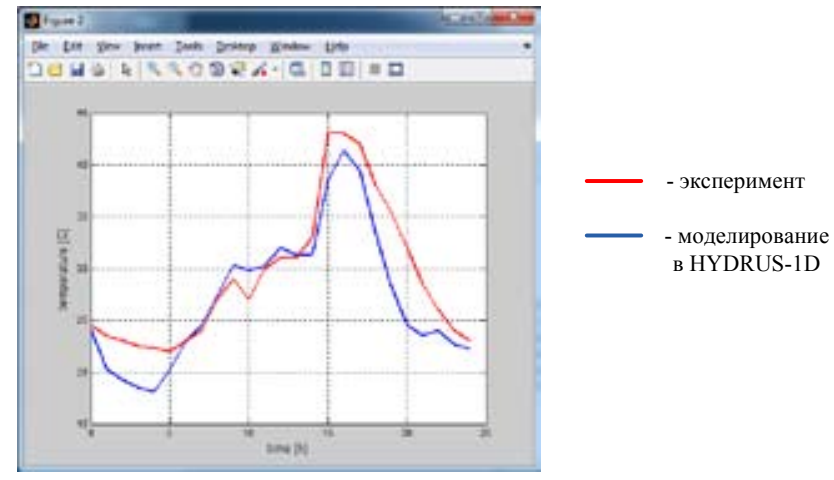

Рис. 3. Распределение температурного поля на поверхности грунта в течение суток

Fig. 3. Distribution of the temperature field on the ground surface within days 
Сравнительный анализ экспериментального и моделируемого распределения температурных полей на поверхности грунта показал, что расчет произведен с достаточной точностью, относительная погрешность мала и составила менее $10 \%$.

\section{Вывод}

Таким образом, произведена постановка задачи на расчет пространственного распределения температурных полей в негомогенной ортотропной переменнонасыщенной среде. Решение данной задачи предлагается с помощью пакета программного обеспечения одномерного моделирования движения воды, тепла и растворов в переменнонасыщенных средах HYDRUS1D с использованием программного обеспечения, созданного в среде MATLAB, позволяющего моделировать суточный ход притока солнечной радиации на поверхность грунта.

В ходе расчета были получены пространственные распределения температур на поверхности и вглубь грунта в течение суток. Точность произведенного расчета подтверждается экспериментальными данными (относительная погрешность составила менее $10 \%$ ), что свидетельствует об адекватности примененной для расчета математической модели.

\section{Работа выполнена при финансовой поддержке РФФИ грант № 18-08-00053 А.}

\section{Список литературы}

[1] Šimůnek J., M. Th. van Genuchten and M. Šejna. The HYDRUS-1D Software Package for Simulating the One-Dimensional Movement of Water, Heat, and Multiple Solutes in Variably-Saturated Media. Version 3.0.J.Šimůnek, M. Th. van Genuchten, M. Šejna. California: Preprint Department of environmental sciences university of California riverside, 2005. 270.

[2] Saito H. Numerical analyses of coupled water, vapor and heat transport in the vadose zone. H. Saito, J. Šimůnek, B. Mohanty. Vadose Zone J. 2006. 5. 784-800.

[3] Семенов М.Е., Аббас 3.Х., Ищук И.Н., Канищева О.И., Мелешенко П.А. Принципы управления обратной связью для перевернутого маятника с гистерезисом в суспензии. Журнал Сибирского федерального университета, Серия: Математика и физика, 2016, 9, 498-509 [Semenov M.E., Abbas Z.H., Ishchuk I.N., Kanishcheva O.I., Meleshenko P.A. Statefeedback control principles for inverted pendulum with hysteresis in suspension. Journal of Siberian Federal University. Mathematics and Physics, 2016, 9 (4), 498-509 (in Russian)].

[4] Ищук И.Н., Филимонов А.М., Степанов Е.А., Постнов К.В. Способ классификации стационарных и квазистационарных объектов по данным динамических инфракрасных изображений, получаемых комплексами с беспилотными летательными аппаратами. Радиотехника, 2016, 10, 145-151. [Ishchuk I.N., Filimonov A.M., Stepanov E.A., Postnov K.V. Method of classification of stationary and nonstationary objects according to the dynamic infrared images obtained complexes with unmanned aerival vehicles. Radiotekchnika, 2016, 10, 145-151 (in Russian)].

[5] Ищук И.Н., Степанов Е.А., Бебенин А.А., Дмитриев Д.Д., Филимонов А.М. Способ классификации объектов оптико-электронными системами разведки на основе обработки многоспектрального кубоида изображений. Журнал Сибирского федерального университета, Серия: Техника и технологии, 2017, 10 (2), 183-190 [Ischuk I.N., Stepanov E.A., Bebenin A.A., Dmitriev D.D., Filimonov A.M. The method of classification of objects by electro-optical intelligence 
systems on the basis of processing of multispectral images of the cuboid. Journal of Siberian Federal University. Engineering and Technology, 2017, 10 (2), 183-190 (in Russian)].

[6] Ищук И.Н., Громов Ю.Ю., Постнов К.В., Степанов Е.А., Тяпкин В.Н. Корреляционная обработка кубоида инфракрасных изображений, получаемых с беспилотных летательных аппаратов. Часть 1. Моделирование и обработка инфракрасных сигнатур техногенных объектов в процессе суточного изменения температур. Журнал Сибирского федерального университета, Серия: Техника и технологии, 2016, 3, 310-318 [Ischuk I.N., Gromov Y.Y., Postnov K.V., Stepanov E.A., Tyapkin V.N. Correlation processing cuboids of infrared images obtained with unmanned aerial vehicles. Part 1. Modeling and processing of infrared signatures of manmade objects in the process of daily temperature change. Journal of Siberian Federal University, Engineering and Technology, 2016, 3, 310-318 (in Russian)]. 